\title{
Source time function and spectra for underground explosions
}

\author{
A R BANGHAR \\ Neutron Physics Division, Bhabha Atomic Research Centre, Trombay, Bombay 400085.
} India

MS received 17 May 1982

\begin{abstract}
Constants occurring in Seggern and Blandford's analytic expression of reduced displacement potential a re computed for shot media of alluvium, salt and tuff. The amplitude spectra as well as impulse response of the source function for models of Haskell and Seggern are studied as a function of yield. It is shown that the maximum in amplitude spectrum shifts to lower frequency with increase in yield for a given model. The duration of impulse response is longer for Seggern's model as compared to Haskell's model for a given yield.
\end{abstract}

Keywords. Source time function: spect ra: underground explosions: displacement potential.

\section{Introduction}

Numerical values of reduced displacement potential are available for a few small underground nuclear explosions detonated in different geological media (Werth and Herbst 1963; Holzer 1966; Rogers 1966: Sisemore et al 1969; Healy et al 1971). Using cube root scaling laws, values of reduced displacement potential were determined for higher yields to synthesize $P$ wave seismograms from underground nuclear explosions (Kogeus 1967, 1968). Haskell (1967) presented analytic expression (involving two arbitrary constants) that fits the numerical values of reduced displacements potential given by Werth and Herbst (1963). This analytic expression made it easy to determine the values of reduced displacement potential at desired intervals of time for various yields. The far-field displacement spectrum derived from analytic expression of Haskell indicates $\omega^{-4}$ frequency dependence at high frequencies and led to unrealistic scaling effects, such as a greater high frequency energy from a small underground explosion than the larger ones (Seggern and Lambert 1970). A model of reduced displacement potential of Mueller and Murphy (1971) gives rise to a far-field displacement spectra that indicates $\omega^{-2}$ dependence at high frequencies and satisfy data in the short-period band recorded teleseismically. Seggern and Blandford (1972) modified Haskell's analytical expression for reduced displacement potential. Seggern and Blandford's analytic expression like Haskell's expression for reduced displacement potential also involves two arbitrary constants. The essential difference in the models of reduced displacement potential of Haskell and Seggern and Blandford is basically in the fall-off in the far-field displacement spectra at high frequencies. Seggern and Blandford (1972) computed the values of two constants by fitting the computed and observed values of reduced displacement potential for granite shot medium. It is equally essential to know the values of these constants for other shot media. We have determined the values of arbitrary constants of Seggern and Blandford analytic 
expression for shot media of salt. tuff and alluvium. It is thus possible to use Seggern and Blandford analytic expression of reduced displacement potential for shot media of salt, tuff and alluvium while synthesizing seismograms.

\section{Source function}

For a spherically symmetric explosive source in an infinite homogeneous medium, the displacement has a radial component, which can be represented in terms of $\psi(\tau)$ as

$$
u(R, t)=-\frac{\partial}{\partial R}(\psi(\tau) / R)
$$

where $\psi(\tau)=$ reduced displacement potential. $\tau=t-R \quad \alpha=$ retarded time,,$=$ tim $c$ $R=$ radial distance from the centre of explosion. $\alpha=$ compressional wave velocity of the medium in which explosion is detonated.

Since $\psi$ is only a function of $\tau$ in the elastic region, its value is independent of $R$. Equation (1) shows that $\psi$ has dimensions of volume and normalls. it is measured in (metre) ${ }^{3}$.

Using (1). it can be shown that

$$
u(R, t)=\psi(\tau) / R^{2}+\frac{1}{\alpha R} \frac{\partial \psi}{\partial \tau}
$$

For large values of $t .(2)$ becomes

$$
R^{2} u(R, \infty)=\psi(\infty)
$$

Equation (3) shows that $\psi(\infty)$ is proportional to permanent displacement.

We are interested in far-field values of the displacement. I'nder this approximation. (2) becomes

$$
u(R . l)=\frac{1}{\alpha R} \frac{\partial}{\partial \tau}(\psi(\tau))
$$

The values of reduced displacement potential $\psi(\tau)$ were calculated by suitable integration of the near-field measurements of velocity and acceleration for a few small underground nuclear explosions in different geological media. The values of reduced displacement potential at desired yields can be computed by using scaling laws (Banghar 1980, 1981).

It was desirable to determine an analytic expression that fits the calculated numerical values of reduced displacement potential. Because, such a relation will be extremely useful for calculating $\psi(\tau)$, at desired intervals of time for explosions of various yields. Haskell (1967) proposed a relation for $\psi(\tau)$ for $\tau$ greater than zero.

$$
\psi(\tau)=\psi(\infty)[1-f(k \tau) \exp (-k \tau)]
$$

where $k$ is a constant to be chosen to fit the time scale of the observed values of $\psi(\tau)$ and $f(k \tau)$ is a polynomial in $k \tau$ to be chosen to match the maximum value of the observed 
$\psi(\tau)$. The minimum permissible degree of $f(k \tau)$ was determined by imposing the conditions that displacement, velocity and acceleration calculated from (5) shall be continuous at $\tau=0$. With these conditions, it can be shown (Haskell 1967), that

$$
f(k \tau)=1+k \tau+\frac{(k \tau)^{2}}{2}+\frac{(k \tau)^{3}}{13}-B(k \tau)^{4},
$$

where $B$ is a dimensionless constant. Values of $B$ and $k$ were determined by fitting the observed and calculated values of $\psi(\tau)$ for underground nuclear explosions of five kilotons yield detonated in granite, salt, tuff and alluvium. Table 1 shows the values of $B . k$ and $\psi(\infty)$ for 5 kilotons explosions in granite. salt, tuff and alluvium. It may be mentioned here that $k$ being inversely proportional to time depends on yield. It scales as inversely proportional to cube root of yield. It is often convenient to discuss the displacement spectrum. Fourier transform of $u(R, t)$ is defined as

$$
u(R, \omega)=\int_{-\infty}^{\infty} u(R . t) \exp (-i \omega t) \mathrm{d} t
$$

Using (4), (5) and (6), it can be shown (Hasegawa 1972; Banghar 1980) that

$$
u(R, \omega)=\frac{\psi(\infty)}{\alpha R}\left[\frac{1+24 B}{[1+i(\omega / k)]^{4}}-\frac{24 B}{[1+i(\omega / k)]^{5}}\right]
$$

where $\omega$ is the angular frequency. Subsequently, we shall refer to the relation given by (8) as Haskell's model.

Equation (8) shows that the far-field displacement spectrum indicates $\omega^{-4}$ dependence at high frequencies. This fall-off indicates a high frequency scaling of spectrum as inversely proportional to the cube root of yield (i.e. large explosions emit less high frequency energy as compared to small ones) and low frequency scaling of displacement proportional to yield (Seggern and Lambert 1970). This frequency scaling of displacement spectrum at high frequencies seems to be unrealistic (Aki et al 1974).

The results of comparing the displacement spectral ratios at one station and first motion amplitudes at several stations for Longshot. Milrow and Cannikin explosions (Seggern and Blandford 1972) indicated that the far-field displacement spectra falls as $\omega^{2}$ at high frequencies. Seggern and Blandford (1972) argued that Haskell's requirement of continuous velocity and acceleration at the elastic radius is too restrictive and

Table 1. Parameters for five kilotons explosions for Haskell's model of the reduced displacement potential in various media.

\begin{tabular}{lccccc}
\hline Medium & $B$ & $K$ & $\psi(\infty)$ & $\begin{array}{c}P \text { wave } \\
\text { velocity } \\
(\mathrm{km} / \mathrm{sec})\end{array}$ & $\begin{array}{l}\text { Density } \\
(\mathrm{g} \mathrm{cm})\end{array}$ \\
\hline Granite & 0.240 & 31.6 & 2500 & 4.80 & 2.69 \\
Salt & 0.171 & 28.4 & 4420 & 4.08 & 2.13 \\
Tuff & 0.050 & 23.5 & 5120 & 2.44 & 1.84 \\
Alluvium & 0.490 & 17.0 & 420 & 1.71 & 1.87 \\
\hline
\end{tabular}


hence can be abandoned. They proposed a model for $\psi(\tau)$ having discontinuous velocity and acceleration at the elastic boundary of the medium surrounding explosion. This was achieved by removing quadric and quartic terms from the expression of $f\left(k_{1} \tau\right)$. The new expression for $\psi(\tau)$ proposed by Seggern and Blandford is given as

$$
\psi(\tau)=\psi(\infty)\left[1-\left(1+k_{1} \tau-B\left(k_{1} \tau\right)^{2}\right) \exp \left(-k_{1} \tau\right)\right]
$$

These investigators determined the values of $B_{1}$ and $k_{1}$ by using the same data as used by Haskell for granite shot medium. We have determined the values of $B_{1}$ and $k_{1}$ for shot media of salt, tuff and alluvium by using values of reduced displacement potential that were used by Haskell (1967). Values of $B_{1}, k_{1}$ and $\psi(\infty)$ are given in table 2 . Reduced displacement potentials derived from expressions (5) and (9) together with the observed reduced displacement potential (Werth and Herbst 1963) for explosions in granite, salt, tuff and alluvium are compared in figures 1 to 4 as a function of retarded time. These figures show that analytic and observed displacement potentials agree closely and the agreement is excellent at very small and very large values of retarded time.

Using (4), (7) and (9), it can be shown that (Banghar 1980)

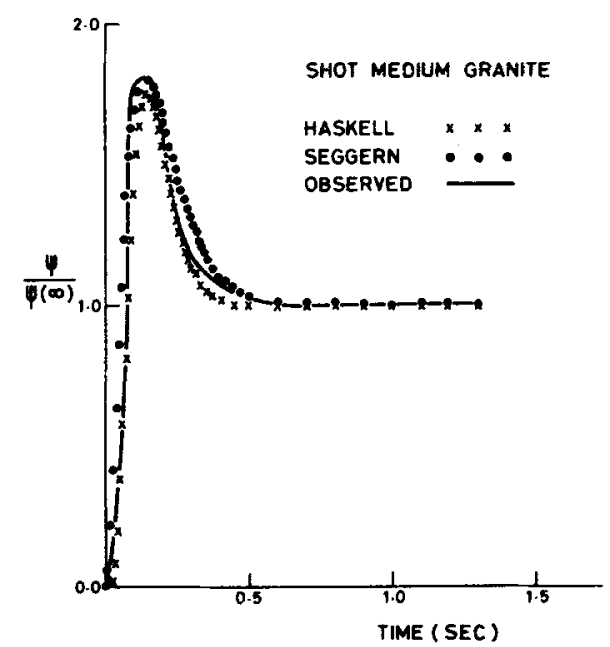

Figure 1. Comparison between observed and analytically derived reduced displacement potentials for 5 kilotons underground nuclear explosions. Analytic expressions used for reduced displacement potential are those of Haskell. Seggern and Blandford.

Table 2. Parameters for 5 kilotons explosions for Seggern and Blandford's model of the reduced displacement potential in various media.

\begin{tabular}{lccccc}
\hline Medium & $B_{1}$ & $K_{1}$ & $\begin{array}{c}\Psi(\infty) \\
\left(\mathrm{sec}^{-1}\right)\end{array}$ & $\begin{array}{c}P \text { wave } \\
\text { velocity } \\
(\mathbf{k m} / \mathbf{s e c})\end{array}$ & $\begin{array}{l}\text { Density } \\
\left(\mathrm{g}^{3} / \mathrm{cm}^{3}\right)\end{array}$ \\
\hline & & & & & \\
Granite & 2.14 & 16.80 & 2500 & 4.80 & 2.69 \\
Salt & 1.43 & 15.60 & 4420 & 4.08 & 2.13 \\
Tuff & 0.35 & 17.02 & 5120 & 2.44 & 1.84 \\
Alluvium & 4.19 & 8.76 & 420 & 1.71 & 1.87 \\
\hline
\end{tabular}




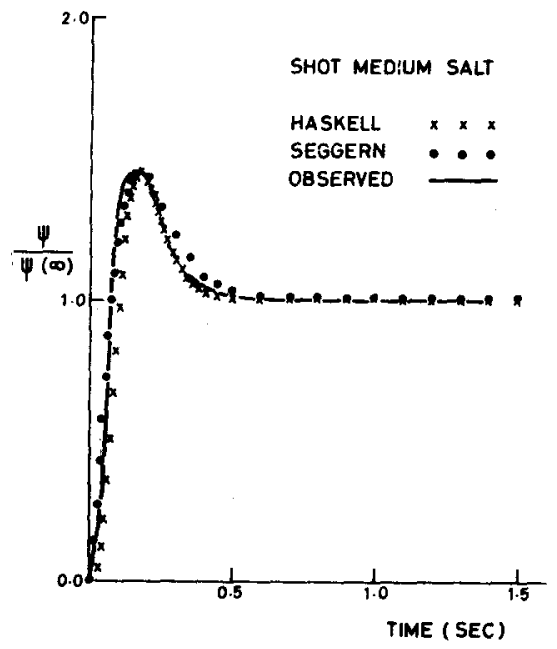

Figure 2. Comparison of observed and analytically derived reduced displacement potentials for 5 kilotons explosions. Models of reduced displacement potential are same as in figure 1 .

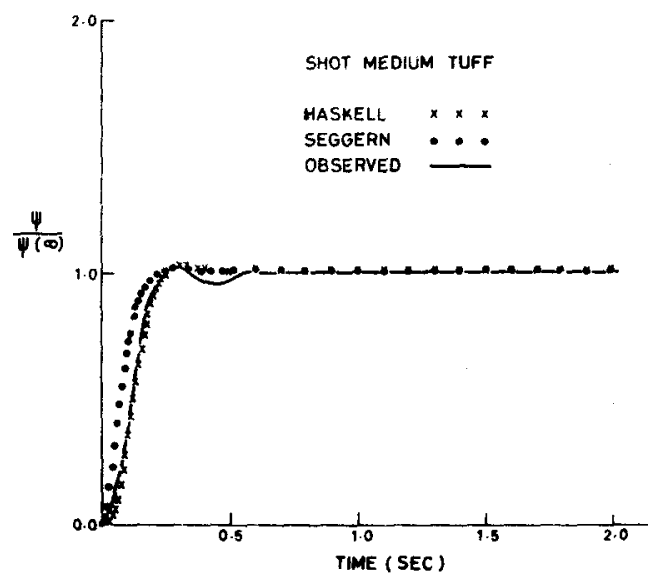

Figure 3. Same as figure 2.

$$
u(R, \omega)=\frac{\psi(\infty)}{\alpha R}\left[\frac{1+2 B_{1}}{\left[1+i\left(\omega / k_{1}\right)\right]^{2}}-\frac{2 B_{1}}{\left[1+i\left(\omega / k_{1}\right)\right]^{3}}\right]
$$

Equation (10) shows that the far-field displacement spectrum falls as $\omega^{-2}$ at high frequencies. This fall-off indicates that far-field displacement spectrum scales proportional to the cube root of yield at high frequencies. The far-field displacement spectrum given by $(10)$ is referred to as Seggern's model subsequently. 


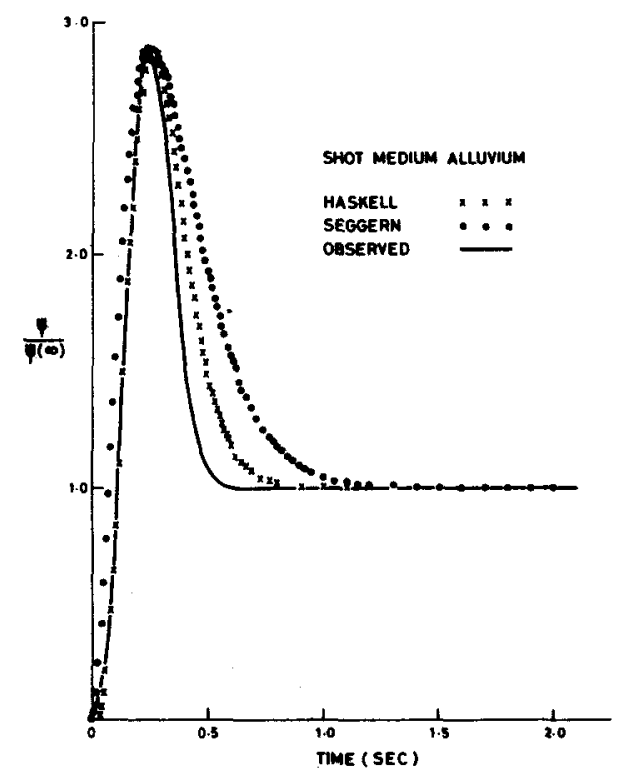

Figure 4. Same as figure 2.

The far-field displacement spectrum (source function) for Haskell and Seggern models given respectively by $(8)$ and $(10)$ is medium as well as yield-dependent. Hence, it is interesting to study the source function as well as its impusle response for both types of these models as a function of yield for various shot media. This study will depict the differences and similarities in the source function and its impulse response due to assumed models. Moreover, an understanding of the behaviour of source function and its impulse response as a function of yield is very useful for synthesizing seismograms from underground nuclear explosions.

Source function for Haskell and Seggern models given respectively by (8) and (10) was computed as a function of frequency for various values of yield for shot media of granite, salt, tuff and alluvium. Figures 5 and 6 show amplitude spectrum (arbitrary units) of source function as a function of frequency for various yields. The assumed source model is indicated at the top in these figures and shot medium is granite. It may be noted that maximum in the amplitude spectrum shifts to a lower frequency with increase in yield for a given model. For example. maximum in amplitude spectrum occurs at 1.8. 0.87 and 0.48 CPS (figure 5) respectively for yields of 10, 100 and 1000 kilotons for Haskell's model. A similar behaviour of source function is observed (figure 6) for Seggern's model also. These figures also show that for a given yield. maximum in the amplitude spectrum occurs at a lower frequency for Seggern's model as compared to Haskell's model.

The impulse response of the source function for these two models was also computed for several yields and the above mentioned shot media. However, impulse response for these two models is depicted (figures 7 and 8 ) as a function of yield only for granite shot media. Figure 7 shows that the duration of the impulse response of the source function increases with the increase in yield. Comparison of figures 7 and 8 indicates that for a given yield, the duration of impulse response of source function is shorter for Haskell's models as compared to Seggern's model. These results suggest 


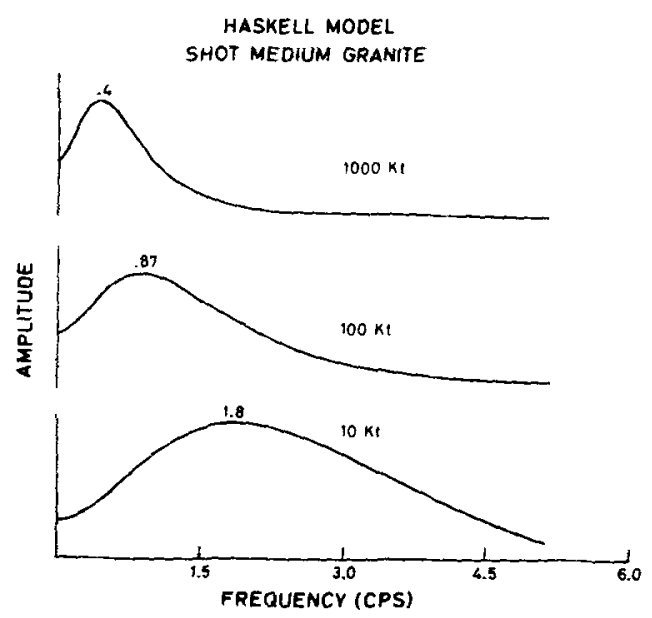

Figure 5. Amplitude spectra of the source function for various values of yield. Source model is that of Haskell and shot medium is granite.

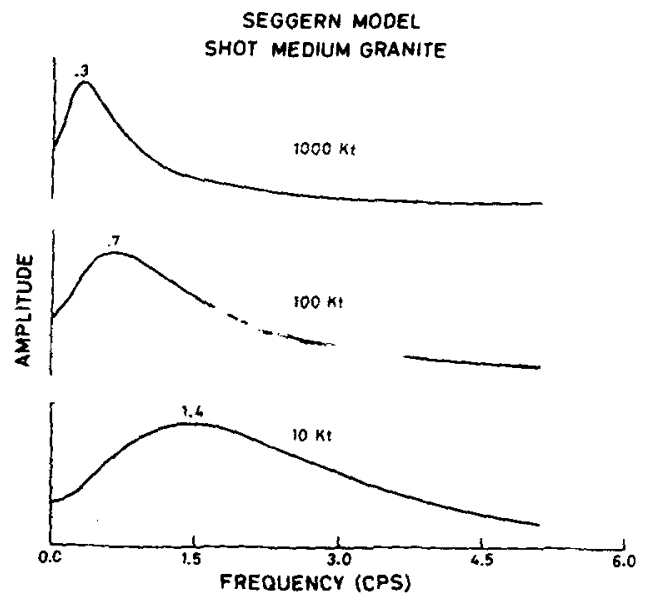

Figure 6. Amplitude spectra of the source function for various values of yield. Source model is that of Seggern and shot medium is granite.

that the period of synthesized $P$ seismograms will depend upon the yield of explosion. Hasegawa (1972) and Springer and Hannon (1973) have presented data that agree with this suggestion.

\section{Discussion and conclusions}

Various constants occurring in analytic expression of reduced displacement potential proposed by Seggern and Blandford were computed for shot media of alluvium, salt and tuff. It is now possible to synthesize $P$ seismograms for these media for source model of Seggern. The variation of spectral amplitude is studied for source models of Haskell and Seggern for different yields. It is observed that the maximum in the spectrum shifts to a lower frequency and the duration of impulse response increases with increase in yield for either of the source models. The duration of impulse response is longer for the Seggern model as compared to the Haskell model for a given yield. 


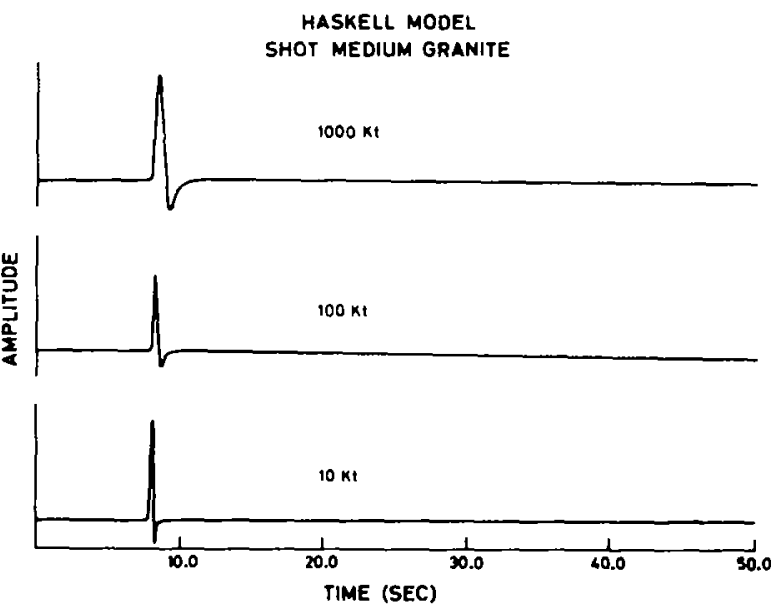

Figure 7. Impulse response of source function for various values of yield. Source model and shot medium are same as in figure 5.
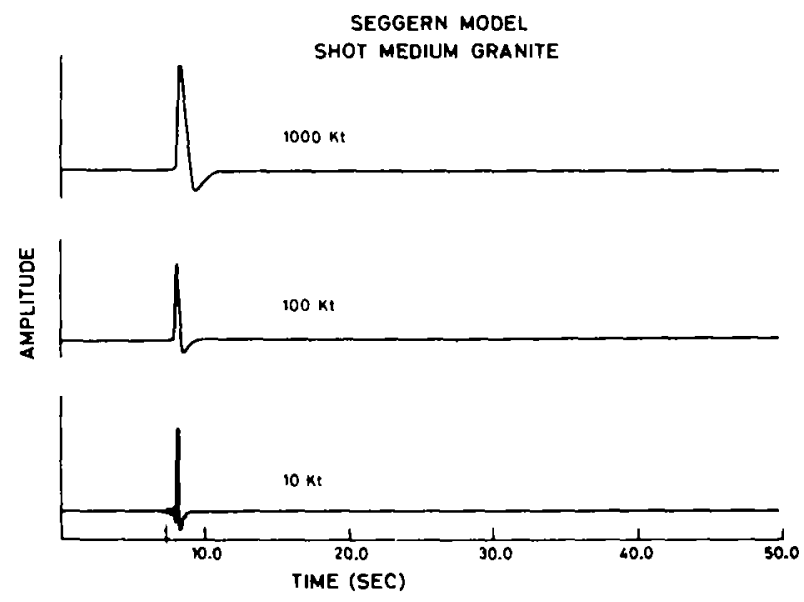

Figure 8. Impulse response of source function for various values of yield. Source model and shot medium are same as in figure 6 .

\section{Acknowledgement}

The author is thankful to I)r (; S Murty for critically reading the manuscript. 


\section{References}

Aki K. Bouchon M and Reasenberg P 1974 Bull. Seism. Soc. Am. 64131

Banghar A R 1980 Synthetic Seismograms - I: Transfer functions for synthesis of $\boldsymbol{P}$ wave seismograms from explosive sources and their parametric study, BARC Report 1-617

Banghar A R 1981 Mechanisms of earthquakes and symthesis of $P$ wave seismograms from explosions and earthquakes, Ph.D. Thesis. Bombay University

Hasegawa H S 1972 J. Geophy's. Res. 773081

Haskell N A 1967 J. Geophys. Res. 722583

Healy J H. King C and O'Neill M E 1971 J. Geophys. Res. 763344

Holzer F 1966 Proc: $R$. Soc. (London) A290 409

Kogeus K 1967 A synthesis of short period $P$ wave records from distant explosion sources. Scientific Report No. 54. Seismological Institute, Uppsala University. Uppsala. Sweden

Kogeus K 1968 Bull. Seism. Soc. Am. 58663

Mueller R A and Murphy J R 1971 Bull. Seism. Soc. Am. 611675

Rogers L A 1966 J. Geophys. Res. 713415

Seggern V D and Lambert D G 1970 J. Geophys. Res. 757385

Seggern V D and Blandford R 1972 Geophy's. J. 3183

Sisemore C J, Rogers L A and Perret W R 1969 J. Geophys. Res. 746623

Springer D L and Hannon W J 1973 Bull. Seism. Soc. Am. 63477

Werth G C and Herbst R F 1963 J. Geophis. Res. 681463 